\title{
$\mathrm{pH}$ 감응성 NIPAM-co-MAA 고분자 마이크로젤의 제조 및 분석과 염 종류에 따른 부피상 변화
}

\author{
Mohammad Saleem Khan ${ }^{\dagger}$, Gul Tiaz Khan, Abbas Khan, and Sabiha Sultana \\ National Centre of Excellence in Physical Chemistry, University of Peshawar \\ (2013년 7월 24일 접수, 2013년 8월 29일 수정, 2013년 9월 4일 채택)
}

\section{Preparation and Characterization of Novel Temperature and pH Sensitive (NIPAM-co-MAA) Polymer Microgels and Their Volume Phase Change with Various Salts}

\author{
Mohammad Saleem Khan ${ }^{\dagger}$, Gul Tiaz Khan, Abbas Khan, and Sabiha Sultana \\ National Centre of Excellence in Physical Chemistry, University of Peshawar, Peshawar-25120, Pakistan \\ (Received July 24, 2013; Revised August 29, 2013; Accepted September 4, 2013)
}

\begin{abstract}
Novel microgels of $N$-isopropylacrylamide (NIPAM)-co-methacrylic acid (MAA) (NIPAM-co-MAA) with different contents of $N, N$-methylene bis acrylamide (MBA) were prepared by emulsion polymerization technique and were studied by Fourier transform infrared spectroscopy (FTIR), dynamic light scattering (DLS) and zeta potential measurement. Effect of $\mathrm{pH}$, temperature and different salts concentration on the microgel particles was investigated. DLS results have shown that the hydrodynamic radius of the microgel increased upon increasing $\mathrm{pH}$ and decreased upon increasing temperature. The swelling/deswelling behaviors as determined by DLS showed the ionic repulsions of the carboxyl group of the methacrylic acid and hydrophobic interaction of NIPAM. The effect of various salts on volume phase transition temperature (VPTT) was also investigated. Upon increasing salt concentration, VPTT became broad and shifted to a lower temperature. Electrophoretic mobility measurements showed an increase with increasing $\mathrm{pH}$ and temperature at a constant ionic strength.
\end{abstract}

Keywords: emulsion polymerization, volume phase transition temperature, swelling/deswelling, electrophoretic mobility, ionic strength.

\section{Introduction}

Among the vast research area in nanoscience, polymer microgels have achieved considerable attention in the polymer science and biomedicine. ${ }^{1,2}$ Among all, microgel particles are considered as large family of crosslinked polymeric nanoparticles; their unique utility is the swelling behavior in a suitable solvent. Hydrogels are three dimensional network structures which swell in water and also in aqueous solutions. Upon swelling these microgels, become soft, rubbery and resemble like living tissues. ${ }^{3}$ Recently, temperature, $\mathrm{pH}$ and other stimuli responsive microgels have made a great deal of attention to scientists. The control of swelling/deswelling prop-

\footnotetext{
${ }^{\dagger}$ To whom correspondence should be addressed.
}

E-mail: saleemkhan@upesh.edu.pk erty is of major technological interest in various areas of medicine, biotechnology and engineering, because swelling/ deswelling can help in detailed knowledge about volume phase transition in microgels. This knowledge can be utilized for developing unique systems for various applications.

An important behavior of a polymer microgel is swelling/ deswelling which depends upon the nature of the solvent used, type of microgel, which further depends on monomer (and/or comonomer) composition/concentration, as well as crosslinker density. The overall properties of the final microgel will be determined from the nature of the monomer used during preparation. Microgels with wide range of different properties can be prepared by the addition of the monomers with different functionalities and makes their possible application range wider. These smart polymers can swell or shrink in response to external stimuli, such as temperature, $\mathrm{pH}$, humidity, ionic 
strength, and solvent composition. ${ }^{4,5}$ Near human body temperature (physiological temperature), poly( $N$-isopropylacrylamide) (PNIPAM) polymer microgels which is temperature responsive undergo reversible volume phase transition (VPT). The temperature responsive PNIPAM-based temperature sensitive microgels makes it popular in various fields which need "smart" material responses to external stimuli, including drug delivery, ${ }^{6}$ optical filtering ${ }^{7}$ and bimolecular recovery. ${ }^{8}$

The utility of such "smart" gels can be broadened by introducing various functionalities in the structure of polymer microgel. The carboxylic acid groups are generally incorporated into PNIPAM gels through the free radical copolymerization of acrylic acid (AA) which is of most particular interest. ${ }^{9}$ Carboxylate groups in MAA depends upon $\mathrm{pH}$, produce an electrophilic position for functional group and increase the volume phase transition temperature of the microgel when present in an ionized state.

Poly (methacrylic acid) (PMAA) hydrogels is an example of polyelectrolyte ones which is sensitive to $\mathrm{pH}$, electric field stimuli and so forth. ${ }^{10}$ The excellent swelling behavior makes them extremely important carriers for drug controlled release. This type of hydrogels can be used as a temperature and $\mathrm{pH}-$ responsive drug deliverers. Microgels having acidic behavior can be utilized for colon specific drug delivery. In stomach, these microgels have low equilibrium degree due to acidic medium and the swelling behavior increases as it goes to the GI (gastrointestinal) tract because of the enhancement in human body $\mathrm{pH}$. The $\mathrm{pH}$ responsive microgels cover the drug from the acidic medium of the stomach and the incorporated/ loaded drug is released in the lower part of the GI tract. ${ }^{11}$ Numerous applications of microgel in various fields including control release and safe drug delivery systems, topical applications i.e., wound dressings, transdermal delivery systems, injectable polymers, dental applications, implants and stimuliresponsive systems. ${ }^{12}$

One of the most important factors that determine their uniqueness is the swelling and deswelling property of the microgels. A number of factors affect the swelling property of stimuli responsible hydrogels, including composition of monomers, crosslinking density, monomers molecular size or crosslinker, nature of crosslinker, ionic strength and $\mathrm{pH}$ of the surrounding media. To modify swelling property of various polymer microgels, these factors are studied.

Here, in this paper novel poly $(\mathrm{N}$-isopropyl acrylamide-comethcrylic acid) [poly(NIPAM-co-MAA)] microgels were prepared through emulsion polymerization and their $\mathrm{pH}$ and tem- perature-sensitive behavior was investigated. Furthermore, the influence of sodium chloride $(\mathrm{NaCl})$, lithium chloride $(\mathrm{LiCl})$ and calcium chloride $\left(\mathrm{CaCl}_{2}\right)$ as a function of temperature on the swelling behavior of microgel was investigated. The zeta potential and electrophoretic mobility of the microgels were measured as function of temperature and $\mathrm{pH}$ at a constant ionic strength. This kind of study has not been reported in literature.

\section{Experimental}

Materials. $\mathrm{N}$-isopropylacrylamide (NIPAM), temperature sensitive monomer, was obtained from Sigma (99\%), purified in acetone-hexane (1:1 volume ratio) mixture, recrystilized and before use dried under vacuum at room temperature. Methacrylic acid (MAA, Sigma, 99.5\%), a pH responsive monomer, ammonium persulfate (APS, Merck), $N, N$-methylene bis-acrylamide (MBA, Sigma), a crosslinker, sodium dodecylsulphate (SDS, Merck), ammonium hydroxide (Merck, 25\%), lithium chloride anhydrous ( $\mathrm{LiCl}$, Merck, 99.5\%), sodium chloride $(\mathrm{NaCl}, \mathrm{Merck}, 99 \%)$, calcium chloride anhydrous $\left(\mathrm{CaCl}_{2}\right.$, Sigma, 99.5\%) and hydrochloric acid ( $\mathrm{HCl}$, Sigma), all were used as obtained from the mentioned suppliers without further purification. Milli-Q deionized water resistance of $18 \mathrm{M} \Omega$ obtained from (Milli-Q Reagent Water System, Millipore Corporation) was used and to remove any particulate matter filtered through a $0.2 \mu \mathrm{m}$ filter.

Preparation of P(NIPAM-co-MAA) Microgels. The microgel particles were prepared by emulsion copolymerization in aqueous solution. In a $250 \mathrm{~mL}$ three necked flask $100 \mathrm{~mL}$ of dust-free deionized water was taken and appropriate amounts of NIPAM, MAA, MBA, and SDS (see Table 1) were added. A three necked glass reactor $(250 \mathrm{~mL})$ contains a stirring glass rod, a $6 \mathrm{~cm}$ Teflon paddle and fitted with a reflux condenser, nitrogen bubbling tube and a septum for initiator injection. The solution was gently heated to $70{ }^{\circ} \mathrm{C}$ in an oil bath to remove oxygen; purging was continued, stirring at $400 \mathrm{rpm}$ for $1 \mathrm{hr}$ was also continued. Then, to start the reaction, $5 \mathrm{~mL}$ APS $(0.0022 \mathrm{~g})$ dissolved in water were injected. The reaction mixture was allowed to proceed for $12 \mathrm{~h}$. Synthesized portion of the novel lattices was further purified from free monomers, SDS and other impurities by three times centrifugations, decantation, and redispersions in Milli-Q deionized water. After these purification processes the microgel were further processed for purification by (Spectra/Por ${ }^{\circledR}$ molecular porous membrane tubing, cutoff 12000-14000) dialysis for four weeks 
Table 1. Compositions of P(NIPAM-co-MAA) Microgel Particles

\begin{tabular}{ccccccccccc}
\hline $\begin{array}{c}\text { Sample } \\
\text { code }\end{array}$ & $\begin{array}{c}\text { MAA } \\
(\mathrm{g})\end{array}$ & $\begin{array}{c}\text { MAA } \\
(\mathbf{\%})\end{array}$ & $\begin{array}{c}\text { NIPAM } \\
(\mathrm{g})\end{array}$ & $\begin{array}{c}\text { MBA } \\
(\mathrm{g})\end{array}$ & $\begin{array}{c}\text { MBA } \\
(\mathbf{\%})\end{array}$ & $\begin{array}{c}\text { SDS } \\
(\mathrm{g})\end{array}$ & $\begin{array}{c}\text { APS } \\
(\mathrm{M})\end{array}$ & Total moles & $\begin{array}{c}\mathrm{H}_{2} \mathrm{O} \\
(\mathrm{mL})\end{array}$ & $\begin{array}{c}\text { Yield } \\
(\mathbf{\%})\end{array}$ \\
\hline $\mathrm{G} 1$ & 0.08298 & $\mathbf{5 \%}$ & 1.87393 & 0.06168 & $\mathbf{2 \%}$ & 0.055 & $\mathbf{1 \times 1 0 ^ { - 3 }}$ & 0.018 & 100 & 91 \\
$\mathrm{G} 2$ & 0.09957 & $\mathbf{6 \%}$ & 1.83319 & 0.12336 & $\mathbf{4 \%}$ & 0.055 & $\mathbf{1} \times 10^{-3}$ & 0.018 & 100 & 90 \\
$\mathrm{G} 3$ & 0.13276 & $\mathbf{8 \%}$ & 1.7721 & 0.1542 & $\mathbf{5 \%}$ & 0.055 & $\mathbf{1 \times 1 0 ^ { - 3 }}$ & 0.018 & 100 & 92 \\
\hline
\end{tabular}

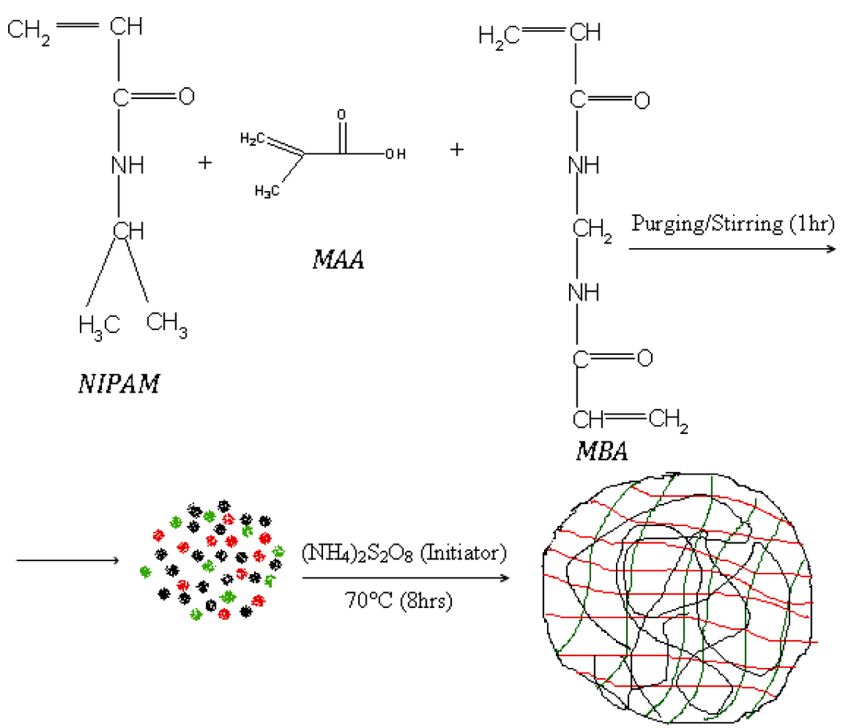

Figure 1. Schematic presentation of microgel synthesis (black color indicates NIPAM, green MAA and red MBA, respectively).

against very frequently changing water at room temperature $\left(25^{\circ} \mathrm{C}\right)$. The remaining microgel obtained from the reaction mixture was centrifuged, decanted, and dried under vacuum to a constant weight at $40{ }^{\circ} \mathrm{C}$ (Figure 1).

Characterization. Fourier transforms infrared spectrophotometer (FTIR) Shimadzu (IR Prestige) Japan was used for the analysis of chemical structures of the pure polymer microgel at a resolution of $4 \mathrm{~cm}^{-1}$ with 100 accumulations. Microgel samples were prepared and the emulsions were dried at $40{ }^{\circ} \mathrm{C}$ for $12 \mathrm{~h}$ and were analyzed using the standard $\mathrm{KBr}$ method.

The experiments were carried out on dynamic light scattering (DLS), a standard 90Plus particle size analyzer (Brookhaven Instruments Corporation, Holtsville, USA) having a detector at $90^{\circ}$. The light source in DLS used was a He-Ne laser $(35 \mathrm{~mW}$, $659 \mathrm{~nm}$ ). For dynamic light scattering (DLS) analysis, the microgel solutions were filtered to remove dust particles through Millipore Millex-HV filters having a pore size of $0.80 \mu \mathrm{m}$. Peltier thermocouple stage was used to keep the temperature of the dispersions constant before DLS analysis in order to maintain at swelling/deswelling equilibrium in water. The temperature of each sample was equilibrated for a min- imum of $20 \mathrm{~min}$.

From DLS measurements, the characteristic line width distribution $G(\Gamma)$ can be obtained from the Laplace inversion of each measured intensity-intensity time correlation function. ${ }^{13}$

Translational diffusion coefficient $D$ has a mathematical relation to the $\Gamma$ for a purely diffusive relaxation, by $\left(\Gamma / q^{2}\right)$ $C \rightarrow 0, q \rightarrow 0=D$, where $q=(4 \pi n / \lambda) \sin (\theta / 2)$ with $\lambda, \theta$ and $n$ is the incident light wavelength in vacuum, the scattering angle, and refractive index of the solvent respectively. StokesEinstein equation

$$
R_{\mathrm{h}}=\left(k_{\mathrm{B}} T / 6 \pi \eta\right) D^{-1}
$$

is used for the transformation of $G(\Gamma)$ into a hydrodynamic radius $\left(R_{\mathrm{h}}\right)$, where $k_{\mathrm{B}}, T$, and $\eta$ are the Boltzmann constant, the absolute temperature, and the solvent viscosity, respectively.

A zeta plus zetameter (Brookhaven Instrument Corp., NJ) was used for the measurements of zeta potential. Polymer microgels surface charge density was determined from the electrophoretic mobility measurements at a temperature (20$55^{\circ} \mathrm{C}$ ) and $\mathrm{pH}(3-10)$. The measurements were carried out as a function of $\mathrm{pH}(3-10)$ at $25^{\circ} \mathrm{C}$. The measurements were performed in a background electrolyte concentration of $1 \times 10^{-3}$ mol dm ${ }^{-3} \mathrm{NaCl}$ (In order to remove variability and to keep constant the length of electrical double layer, background electrolyte is used) solution. Samples solution $\mathrm{pH}$ was adjusted with $0.1 \mathrm{M} \mathrm{HCl}$ and $0.1 \mathrm{M} \mathrm{NaOH}$. The microgel samples were diluted within the operating range of the machine in order to get fruitful readings ( 1 in 5 dilutions) using the background $\mathrm{NaCl}$ solution. The electrophoretic mobility values were the average value of the 10 readings (or transformed into $\hat{e}$ potential) at a fixed $\mathrm{pH}$ value.

\section{Results and Discussion}

FTIR Study of P(NIPAM-co-MAA) Microgel. The characteristic peaks for the copolymers (G1, G2, and G3) are observed as shown in Figure 2. The peak at $1728 \mathrm{~cm}^{-1}$ is for the $\mathrm{C}=\mathrm{O} ; 1461 \mathrm{~cm}^{-1}$ is ascribed to the $\mathrm{C}-\mathrm{N}$ stretching of the 


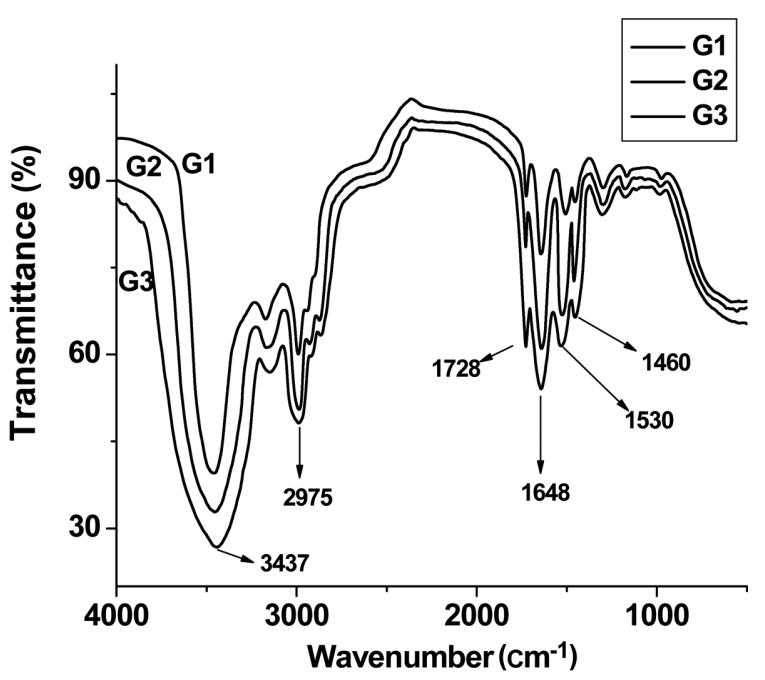

Figure 2. FTIR spectra of P(NIPAM-co-MAA) (G1, G2, G3) microgels.

amide bond of G1, G2, and G3; the peak around $1530 \mathrm{~cm}^{-1}$ is due to the $\mathrm{N}-\mathrm{H}$ stretching of the amide bond. The peaks around 1366 and $1388 \mathrm{~cm}^{-1}$ are for two methyl groups deformation on $\left.-\mathrm{C}\left(\mathrm{CH}_{3}\right)_{2}\right) ; 1460 \mathrm{~cm}^{-1}$ is for the $-\mathrm{CH}_{3}$ and $-\mathrm{CH}_{2}-$ deformation; $1551 \mathrm{~cm}^{-1}$ peak for secondary amide N-H stretching of amide II band and $1648 \mathrm{~cm}^{-1}$ peak is for amide I band. The peaks at $2877 \mathrm{~cm}^{-1}$ are $-\mathrm{CH}_{3}$ symmetric stretching; 2934 $\mathrm{cm}^{-1}$ peak is for asymmetric $-\mathrm{CH}_{2}$ - stretching; $2975 \mathrm{~cm}^{-1}$ peak is $-\mathrm{CH}_{3}$ asymmetric stretching; $3437 \mathrm{~cm}^{-1}$ peak is N-H stretching, which is in accordance with PNIPAM. ${ }^{14}$

The appearance of the characteristic absorption band of carboxyl group in curve G1, G2, and G3 of Figure $2\left(1728 \mathrm{~cm}^{-1}\right)$ is a strong evidence of incorporation of MAA into the copolymer microgel.

Dynamic Laser Light Scattering Studies on P(NIPAMco-MAA) Microgel Series. Effect of Temperature P(NIPAMco-MAA) Microgel Series: DLS measurements were used to judge the hydrodynamic radius in the temperature range of 20$70{ }^{\circ} \mathrm{C}$ of the polymer microgels at $\mathrm{pH}(3-12)$. Hydrodynamic radius of the polymer microgel particles is dependent upon temperature. Since poly(NIPAM) based microgels are temperature sensitive, they show deswelling above $33^{\circ} \mathrm{C}$. Thermosensitive polymeric microgels swelling and de-swelling behavior depends upon different types of polymer-polymer and polymer-solvent interactions. These include: ionic interactions, hydrogen bonding, hydrophobic interactions, and Van der Waals interactions. At $20^{\circ} \mathrm{C}$ poly(NIPAM) particles have a very low Hamaker constant (Van der Waal interactions) and are swollen with water similar to that of the solvent. Due to low

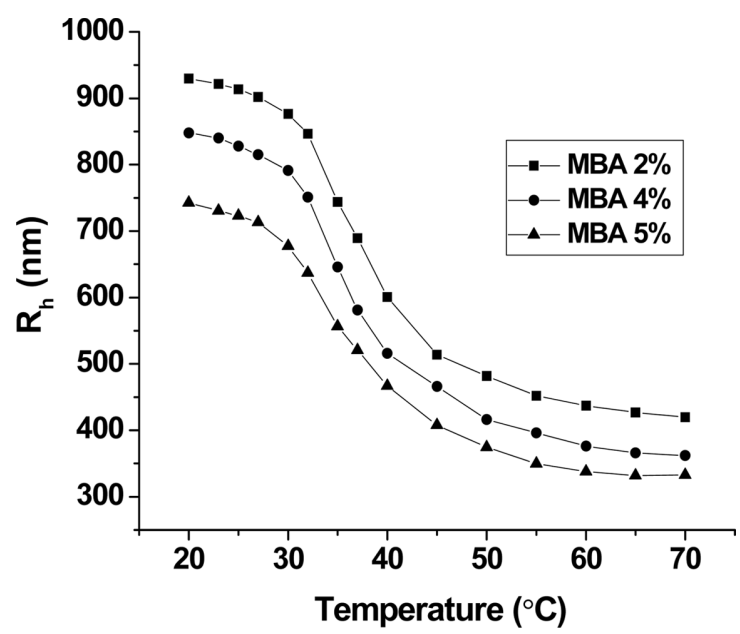

Figure 3. Hydrodynamic radius of P(NIPAM-co-MAA) microgel as a function of temperature at $\mathrm{pH}$ 6.5.

Van der Waals attractive forces among the microgel particles it makes stable dispersions by making hydrogen-bonded structure with bulk water molecules and with interstices. By increasing temperature, contraction of the microgel particles takes place which results in the elimination of water from the interstitial spaces of the microgels and the polymer chain becomes more hydrophobic and polymer-solvent interactions are minimized. ${ }^{15}$

From Figure 3 it is clear that hydrodynamic radius of the microgels containing 5 and 8 mol\% MAA (2, 4 and 5\% MBA) is dependent on temperature at $\mathrm{pH} 6.5$ and underwent shrinking transition in the temperature range of $30 \sim 35^{\circ} \mathrm{C}$. It is seen that the shrinking transition was broader and shifted to higher temperature upon increasing the degree of crosslinking. Above the lower solution critical temperature (LCST), however, the swelling behavior decreased. At low temperatures, the microgel particles had a higher swelling ratio because the NIPAM component was swellable at a temperature lower than the LCST. By increasing temperature, dehydration occurred in the NIPAM network, which results in subsequent aggregation of the NIPAM chains and causes deswelling of the mircrogels. ${ }^{16}$

Effect of $\mathrm{pH}$ on P(NIPAM-co-MAA) Microgel Series. The hydrodynamic radius of the microgel particles depends upon $\mathrm{pH}$ (at a constant temperature of $22^{\circ} \mathrm{C}$ ). By plotting the change in the hydrodynamic radius of the microgels $v s . \mathrm{pH}$, DLS data shows that MAA strongly depends upon $\mathrm{pH}$ and plays an important role in the microgel swelling and deswelling behavior. From Figure 4 it is clear that the shrinking phenomenon as well as the size of the microgels depends strongly 


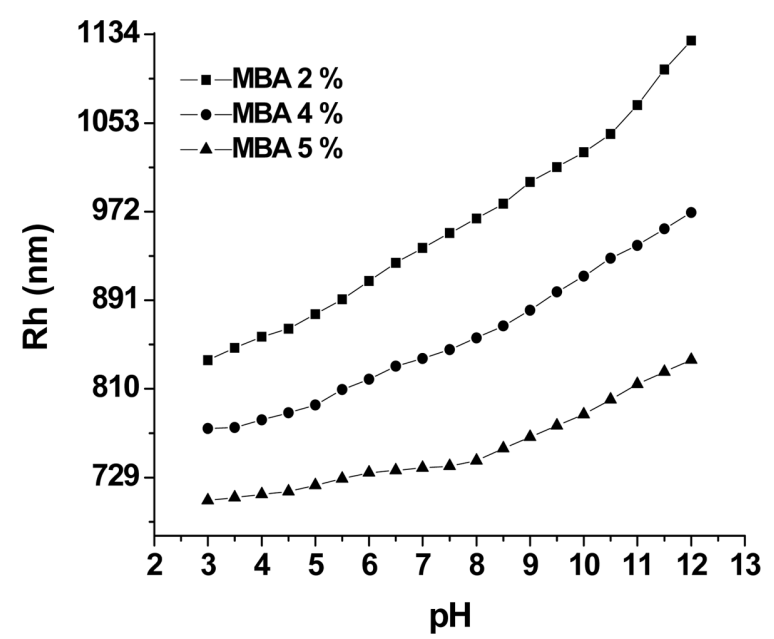

Figure 4. Hydrodynamic radius of P(NIPAM-co-MAA) microgels as a function of $\mathrm{pH}$ at $22^{\circ} \mathrm{C}$.

on $\mathrm{pH}$. By increasing $\mathrm{pH}$ methacrylic acid groups dissociates releasing proton and results in the increase the charge density on the polymer chain. In the swollen state, i.e at low temperature, by increasing $\mathrm{pH}$ the hydrodynamic radius of the microgel particles increases. By increasing $\mathrm{pH}$ the inter ionic repulsion of the carboxylic groups increases because of the increase concentration of carboxylate groups due to dissociation with $\mathrm{pH}$, which shows that at low temperature swelling strongly depends on $\mathrm{pH}$ [9]. Hydrodynamic radius of the microgels containing $8 \mathrm{~mol} \%$ of MAA decreased upon increasing the concentration of MBA. There was not much difference in the degree of swelling below $\mathrm{pH} 5$, but the microgel particles greatly swelled above $\mathrm{pH}$ 5. From Figure 4 it is clear that the gel having MBA concentration $2 \%$ has higher swelling behavior with $\mathrm{pH}$ as compared to those having $4 \%$ and $5 \%$ MBA due to more crosslinking behavior of MBA.

Swelling (or deswelling) ratio $(\alpha)$ is defined as the volume ratio before and after phase transition, which can be calculated with the following equation. ${ }^{17}$

$$
\alpha=\left[\frac{R h 20^{\circ} \mathrm{C}}{R h 70{ }^{\circ} \mathrm{C}}\right]^{3}
$$

Here, swelling (or deswelling) ratio $(\alpha)$ were investigated by the ratio of the microgel size measured by DLS at $20^{\circ} \mathrm{C}$ to that at $70{ }^{\circ} \mathrm{C}$.

Generally the degree of swelling of the microgels increased under basic conditions. The pK $\alpha$ of PMAA is 5.5 ; therefore the carboxylic groups in PMAA are not completely dissociated below $\mathrm{pH} 5.5$ which strengthens hydrogen bonding chances occurring between carboxylic groups of PMAA and amide

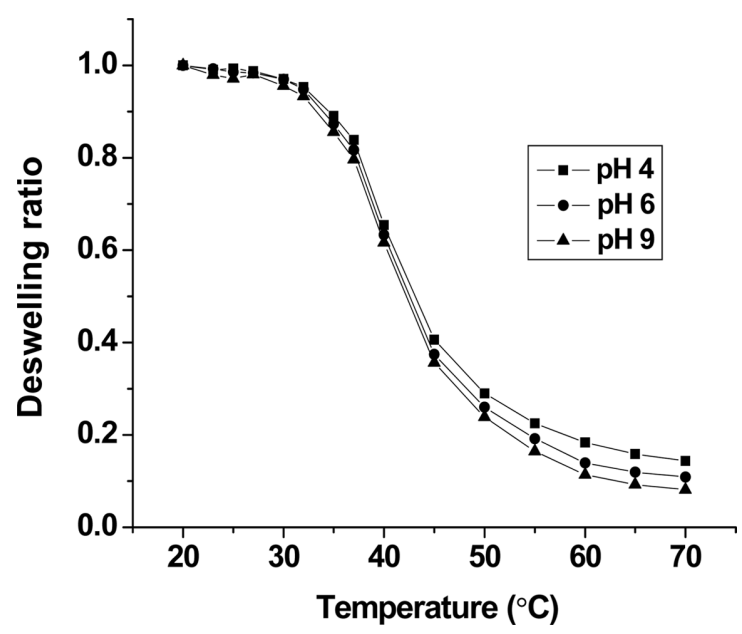

Figure 5. Deswelling ratio of $\mathrm{P}(\mathrm{NIPAM}-\mathrm{co}-\mathrm{MAA})$ microgels at different $\mathrm{pH}$ at $22^{\circ} \mathrm{C}$.

groups of NIPAM. ${ }^{18}$ The carboxylate groups of microgel particles are highly in dissociated form at a pH above 5.5 and as a result expansion of the microgel particles occurs due to internal electrostatic repulsion between the negative charges. From Figure 5 we found that with increasing $\mathrm{pH}$ the swelling ratio increased (deswelling ratio decreased) due to strong inter ionic repulsion of the carboxylic acid side chains and the ionic osmotic pressure caused by the mobile counterions of the charged ions in the network. ${ }^{19}$

Effect of MBA Concentration on the Swelling Behavior. Due to presence of cross-linker mainly MBA, the swelling behavior of polymer microgel is limited. Figure 6 shows the deswelling ratio of microgel with varying MBA concentration $v s$. temperature for a series of microgels. As expected, the

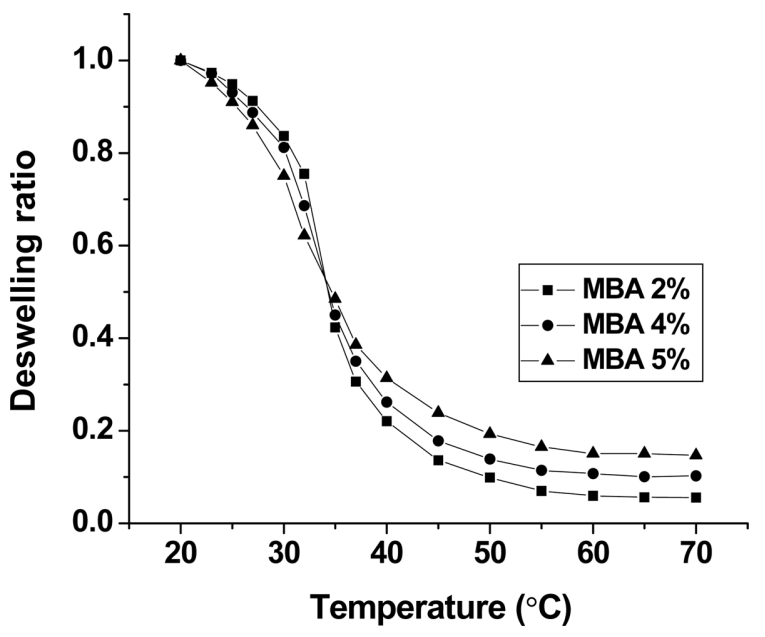

Figure 6. The influence of MBA content on microgel swelling at $\mathrm{pH} 6$ and $22^{\circ} \mathrm{C}$. 
deswelling ratios increased with increasing MBA content. The smallest particle size at high temperature is due to lowest concentration of cross-linker MBA but it gives the maximum particle concentration. ${ }^{20}$

Effect of Different Salts on the Swelling/Deswelling of P(NIPAM-co-MAA) Microgel Series. Ionic strength plays an important role in microgels swelling behavior. Therefore, the influence of the ionic strength on the swelling behavior was investigated at different salts concentrations. Generally, increasing ionic strength reduces inter ionic repulsive forces among the dissociated carboxylate groups and methacrylic acid and decreases the debye screening length. When salts are added, the ionic strength varies, so, we conclude that the salt solution have an important role in the swelling behavior. ${ }^{21}$

The sizes of microgels calculated for different salts at different temperatures are given in Table 2 .

The swelling ratio of these three gels was measured in different salt solutions from Table 2 and given in Figure 7 for the three salts which we have studied. From the deswelling ratio figures, the cation effect on the swelling behavior can be visualized. Upon increasing cation charge, the crosslinking density is increased which consequently decreases the swelling. Therefore, synthesized hydrogel absorbency was found to be in the order of $\mathrm{NaCl}>\mathrm{LiCl}>\mathrm{CaCl}_{2}$.

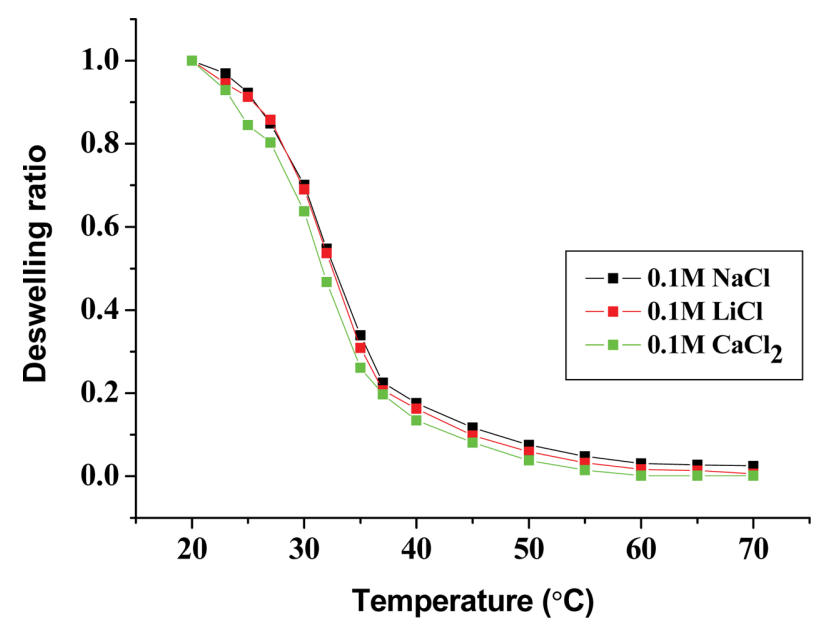

Figure 7. Deswelling ratio of G1 microgel vs. temperature at constant ionic strength of various salts $\left(\mathrm{NaCl}, \mathrm{LiCl}\right.$ and $\left.\mathrm{CaCl}_{2}\right)$ at $\mathrm{pH} 6$.

The influence that temperature has on the swelling behavior of the microgel containing 2\% MBA at different ionic strength of $\mathrm{NaCl}$ and $\mathrm{pH} 6$ is shown in the Figure 7.

In the presence of concentrated salt solution, the deswelling transition of the microgel particles occurred in a broad temperature range. The deswelling ratio of the microgel particles decreased upon increasing ionic strength; this effect is due to the shielding effect caused by the counterions at higher ionic

Table 2. Particle Sizes of Microgels at Different Temperatures for Different Salts

A: $\mathrm{NaCl}$

\begin{tabular}{lccccccccccccccc}
\hline \multicolumn{110}{c}{ Size in nanometer at different temperature $\left({ }^{\circ} \mathrm{C}\right)$} \\
\hline Conc. $(\mathrm{M})$ & 20 & 23 & 25 & 27 & 30 & 32 & 35 & 37 & 40 & 45 & 50 & 55 & 60 & 65 & 70 \\
\hline 0.001 & 300 & 297 & 295 & 293 & 290 & 285 & 266 & 247 & 209 & 186 & 146 & 137 & 121 & 119 & 113 \\
0.01 & 277 & 276 & 273 & 267 & 259 & 246 & 222 & 189 & 167 & 150 & 125 & 113 & 104 & 99 & 97 \\
0.1 & 257 & 254 & 249 & 242 & 227 & 209 & 179 & 152 & 142 & 125 & 108 & 103 & 94 & 91 & 89 \\
\hline
\end{tabular}

B: $\mathrm{LiCl}$

\begin{tabular}{lccccccccccccccc}
\hline \multicolumn{11}{c}{ Size in nanometer at different temperature $\left({ }^{\circ} \mathrm{C}\right)$} \\
\hline Conc. (M) & 20 & 23 & 25 & 27 & 30 & 32 & 35 & 37 & 40 & 45 & 50 & 55 & 60 & 65 & 70 \\
\hline 0.001 & 293 & 288 & 285 & 282 & 276 & 269 & 247 & 218 & 191 & 168 & 138 & 130 & 115 & 112 & 105 \\
0.01 & 269 & 266 & 263 & 258 & 250 & 237 & 205 & 179 & 161 & 137 & 116 & 107 & 97 & 92 & 90 \\
0.1 & 243 & 240 & 237 & 231 & 214 & 197 & 164 & 143 & 132 & 113 & 99 & 92 & 85 & 83 & 81 \\
\hline
\end{tabular}

$\mathrm{C}: \mathrm{CaCl}_{2}$

Size in nanometer at different temperature $\left({ }^{\circ} \mathrm{C}\right)$

\begin{tabular}{|c|c|c|c|c|c|c|c|c|c|c|c|c|c|c|c|}
\hline Conc. (M) & 20 & 23 & 25 & 27 & 30 & 32 & 35 & 37 & 40 & 45 & 50 & 55 & 60 & 65 & 70 \\
\hline 0.001 & 283 & 278 & 271 & 269 & 261 & 247 & 230 & 204 & 182 & 154 & 137 & 117 & 106 & 102 & 99 \\
\hline 0.01 & 258 & 251 & 249 & 242 & 231 & 216 & 182 & 168 & 139 & 122 & 103 & 94 & 88 & 84 & 83 \\
\hline 0.1 & 228 & 223 & 216 & 212 & 201 & 177 & 146 & 134 & 122 & 108 & 89 & 81 & 76 & 74 & 72 \\
\hline
\end{tabular}


strength. The continuous transition occurred in the temperature range $20-70{ }^{\circ} \mathrm{C}$ with continuous transition in $10^{-3}-10^{-1} \mathrm{M} \mathrm{NaCl}$ solution. It is obvious that the continuous variation of the hydrodynamic radius is not due to coagulation.

Figure 7 also shows the deswelling ratio of the microgel for lithium chloride concentration. From these results we concluded that when the salt concentration increases, the swelling ratio is decreased. At low salt concentration, the concentration of ions within the microgel structure exceeds the concentration of salt in the external solution, and the microgel expands due to large ion-swelling pressure. It means that initially there is scarcity of ions inside the microgels which favors inside movement of ions. The ions from low salt concentrated solutions enter there, resulting in expansion. But when salt concentration is high, the movement between the internal and external ion decreases which results in the shrinkage of the hydrogel. At higher ionic strength the hydrogel continues to shrink until the mobile-ion swelling pressure inside and outside becomes approximately equal. The shrinkage phenomenon can also be studied on the basis of electrostatic repulsion of the carboxylate groups in the microgel. By increasing salt concentration, inter ionic repulsion is decreased and the microgels shrink. (Charge screening effect). ${ }^{22}$

NIPAM-MAA microgels electrophoretic mobility of the strongly depends upon $\mathrm{pH}$ which is shown in Figure 8. The electrostatic repulsion among the charged groups caused by deprotonation of the MAA carboxylic groups and due to mobile counterions osmotic contribution into the microgel network, and a Donnan equilibrium driven swelling occurs. At higher ionic strength, microgel swelling is lower because the

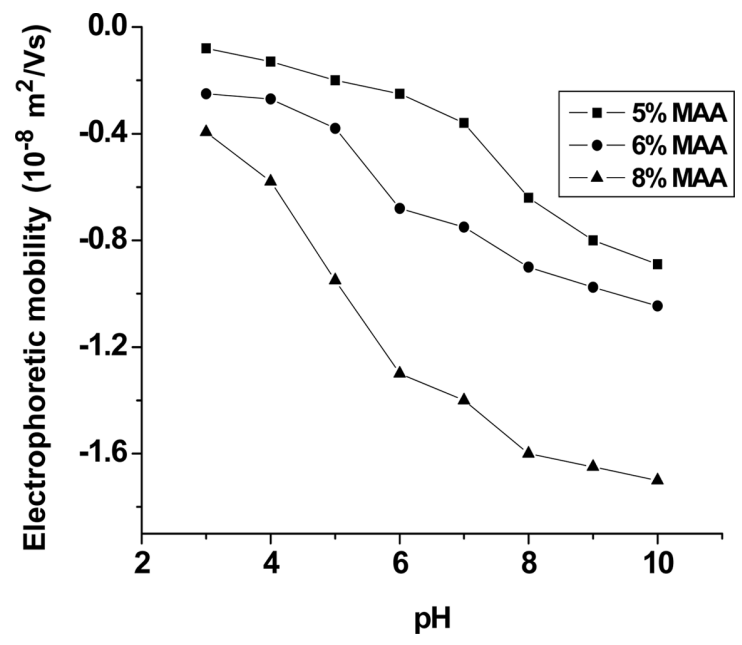

Figure 8. Electrophoretic mobility of P(NIPAM-co-MAA) microgel vs. $\mathrm{pH}$ at $25^{\circ} \mathrm{C}$ and a constant ionic strength: $1 \times 10^{-3} \mathrm{M} \mathrm{NaCl}$. presence of higher concentration of salt causes charge shielding of the carboxylate ions. Furthermore, at a $\mathrm{pH}$ below the MAA pK $\alpha(\sim 5-6)$, the microgel shows a clear temperaturesensitive swelling behavior, whereas at values above that $\mathrm{pH}$ range the hydrodynamic radius differences at 24 and $37^{\circ} \mathrm{C}$ were not significant. ${ }^{23}$

The electrophoretic mobility of P(NIPAM-co-MAA) microgels, measured as a function of $\mathrm{pH}$ and temperature in $1 \times 10^{-3}$ $\mathrm{M} \mathrm{NaCl}$ solution, are shown in Figures 8 and 9. The microgel particles exhibited negative values for these parameters, which both decreased upon increasing the $\mathrm{pH}$ and temperature.

The electrophoretic mobility and zeta potential of the particles containing $10 \mathrm{~mol} \%$ MAA was much lower relative to

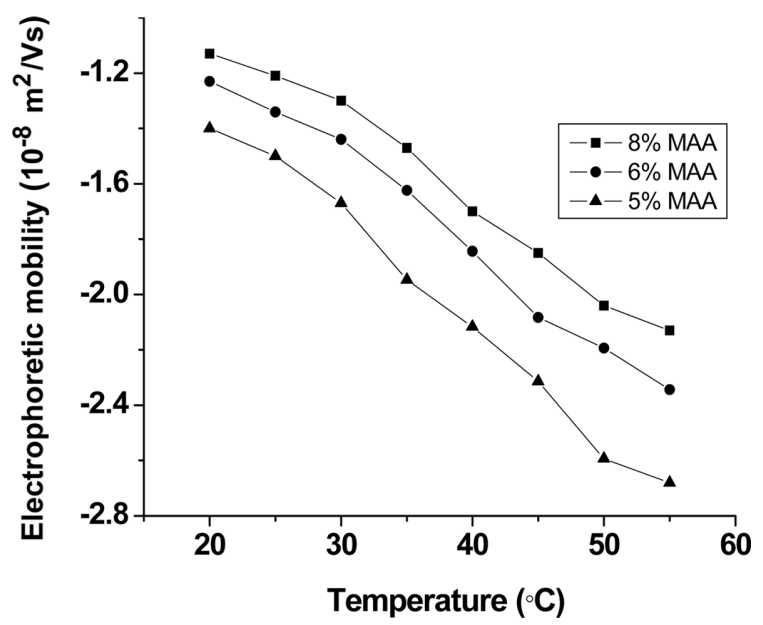

Figure 9. Electrophoretic mobility of P(NIPAM-co-MAA) microgel $v s$. temperature at $\mathrm{pH} 6$ and a constant ionic strength: $1 \times 10^{-3} \mathrm{M}$ $\mathrm{NaCl}$.

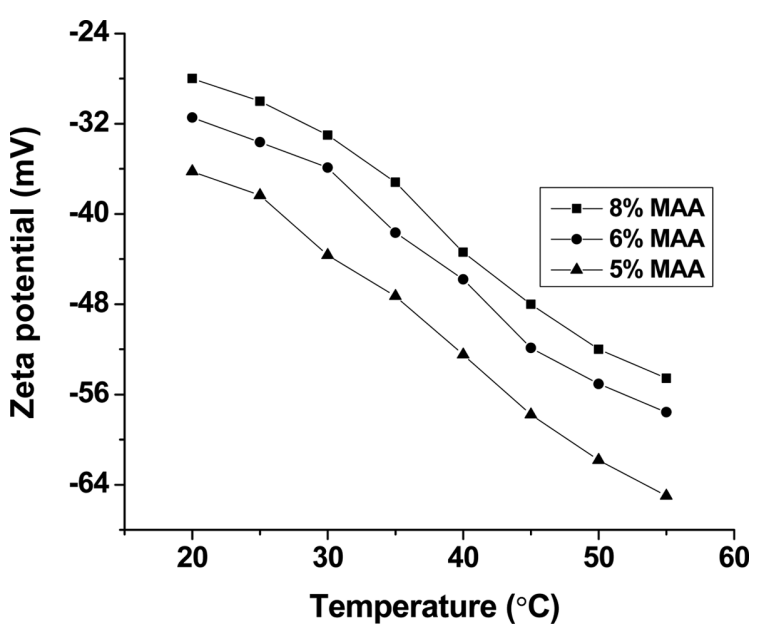

Figure 10. Zeta potentials of P(NIPAM-co-MAA) microgel $v s$. temperature at $\mathrm{pH} 6$ and a constant ionic strength: $1 \times 10^{-3} \mathrm{M} \mathrm{NaCl}$. 
those containing $6 \%$ and either 2 or $4 \%$ MBA. This finding reflects the higher surface charge density imparted by the presence of more MAA. The observed negative electrophoretic mobility and zeta potential for the microgel particles as shown in Figure 10 is due to the increasing surface charge density caused by the dissociation of carboxylic acid groups upon increasing the $\mathrm{pH}^{24}$

The electrophoretic mobility for the microgel particles also decreased above the LCST; as shown in Figure 9. This effect was caused by shrinkage of the hydrogel layer, which induces in enhances in the surface charge density. ${ }^{16}$

\section{Conclusions}

In this study, P(NIPAM-co-MAA) microgels were prepared by emulsion polymerization using MBA as a crosslinker. The obtained novel microgels showed both temperature and $\mathrm{pH}$ sensitivity. The microgel hydrodynamic radius containing different MBA contents were distributed around 350-950 nm. Above $\mathrm{pH} \mathrm{5,} \mathrm{the} \mathrm{microgel} \mathrm{particles} \mathrm{were} \mathrm{greatly} \mathrm{swollen.} \mathrm{The}$ shrinking transition of the microgel particles occurred in a temperature range of $30-37^{\circ} \mathrm{C}$.

The swelling ratio of the microgel particle decreased drastically as temperature, ionic strength and $\mathrm{pH}$ increased. By increasing temperature and $\mathrm{NaCl}$ concentration, swelling ratio decreased. The swelling studies of the novel polymer microgels in various salt solutions showed better swelling behavior, especially in monovalent cations solutions. However, swelling loss in salt solutions can be attributed to a charge screening effect and ionic crosslinking for mono- and multi-valent cations, respectively. The swelling degree in $\mathrm{CaCl}_{2}$ was much lower than that in $\mathrm{LiCl}$ and $\mathrm{NaCl}$ solution and distillated water.

The electrophoretic mobility and zeta potential of the microgel particles had negative values that decreased drastically upon increasing $\mathrm{pH}$ and temperature. We concluded that the increase in the surface charge density was induced by dissociation of the carboxylic acid groups and shrinkage of the particle sizes of the microgels.

Acknowledgement: One of us (AK) acknowledges the financial support of Higher Education Commission, Islamabad, Pakistan under the IPFP program.

\section{References}

1. A. K. Andrianov and L. G. Payne, Adv. Drug Deliv. Rev., 34, 155 (1998).

2. S. V. Vinogradov, T. K. Bronich, and A. Kabanov, Adv. Drug Deliv. Rev., 54, 135 (2002).

3. N. A. Peppas, P. Bures, W. Leobandung, and H. Ichikawa, Eur. J. Pharm. Biopharm., 50, 27 (2000).

4. M. Bradley, J. Ramos, and B. Vincent, Langmuir, 21, 1209 (2005).

5. M. J. Murray and M. J. Snowden, Adv. Colloid Interface Sci., 54, 73 (1995).

6. P. F. Kiser, G. Wilson, and D. Needham, Nature(London), 394, 459 (1998).

7. J. M. Weissman, H. B. Sunkara, A. S. Tse, and S. A. Asher, Science, 274, 959 (1996).

8. E. L. Cussler, M. R. Stokar, and J. E. Vararbert, AIChE J., 30, 578 (1984).

9. K. Kratz, T. Hellweg, and W. Eimer, Colloid Surface A, 170, 137 (2000).

10. J. Ostroha, M. Pong, A. Lowman, and N. Dan, Biomaterials, 25, 4345 (2004).

11. H. Brondsted and J. Kopecek, Biomaterials, 12, 584 (1991).

12. N. A. Peppas and A. G. Mikos, Hydrogels in Medicine and Pharmacy, CRC Press, Boca Raton, FL, Vol. 01, pp 1-25 (1986).

13. G. Z. Zhang, M. Jiang, L. Zhu, and C. Wu, Polymer, 42, 151 (2001).

14. Y. V. Pan, R. A. Wesley, and R. Luginbuhl, Biomacromolecules, 2, 32 (2001).

15. H. Nur, V. T. Pinkrah, J. C. Mitchell, L. S. Benee, and M. J. Snowden, Adv. Colloid Interface Sci., 158, 15 (2010).

16. K. S. Kim, M. H. Kim, and S. H. Cho, J. Ind. Eng. Chem., 11, 736 (2005).

17. Y. Zhang, T. Liu, Q. Wang, J. Zhao, J. Fang, and W. Shen, Macromol. Res., 20, 484 (2012).

18. V. C. Chan and A. S. Hoffmann, Nature, 373, 49 (1955).

19. T. Tanaka, D. Fillmore, S. Sun, I. Nishio, and G. Swislow, Phys. Rev. Lett., 45, 1636 (1980).

20. M. Sadeghi and N. Ghasemi, Indian J. Sci. Technol., 5, 1879 (2012).

21. M. Sadeghi and H. Hosseinzadeh, Turk. J. Chem., 34, 739 (2010).

22. M. Sadeghi, Braz. J. Chem. Eng., 29, 295 (2012).

23. E. Costa, M. M. Lloyd, C. Chopko, A. R. Ana, and P. T. Hammond, Langmuir, 28, 10082 (2012).

24. T. Hoare and R. Pelton, Macromolecules, 37, 2544 (2004). 